\title{
Novas cerâmicas ferroelétricas transparentes com altos coeficientes eletroópticos: PLMN-PT
}

\author{
(New transparent ferroelectric ceramics with \\ high electro-optical coefficients: PLMN-PT)
}

\author{
F. A. Londoño, J. A. Eiras, D. Garcia \\ Grupo de Cerâmicas Ferroelétricas - GCFerr, Departamento de Física, Universidade Federal de S. Carlos, \\ Rod. Washington Luiz, km 235, S. Carlos, SP 13565905 \\ flondono@df.ufscar.br,eiras@df.ufscar.br,ducinei@df.ufscar.br
}

\begin{abstract}
Resumo
Cerâmicas de niobato de magnésio e chumbo (PMN) pertencem à classe de materiais ferroelétricos usados em diversas aplicações tecnológicas. Contudo, existe grande dificuldade em produzir cerâmicas de PMN sem a formação de fases secundarias. Estudos recentes mostraram que a adição de PT e La permitem, respectivamente, estabilizar a fase perovskita e atingir-se a transparência da ordem das cerâmicas comerciais de PLZT. Neste trabalho realizou-se a síntese de pós do sistema (1-x)PLMN-xPT em função de PT $(11 \leq \mathrm{x} \leq 15)$, para $1 \% \mathrm{em} \mathrm{mol} \mathrm{de} \mathrm{La.} \mathrm{Encontraram-se} \mathrm{apenas} \mathrm{traços} \mathrm{de} \mathrm{fase} \mathrm{pirocloro} \mathrm{(2-3 \% )} \mathrm{nos} \mathrm{difratogramas} \mathrm{de} \mathrm{raios} \mathrm{X} \mathrm{dos} \mathrm{pós,}$ sem uma relação direta com a concentração de PT. Foram alcançadas alta taxa de densificação e microestrutura homogênea com prensagem uniaxial a quente. A transmitância óptica passou por um máximo (até $55 \% \mathrm{em} \lambda=632 \mathrm{~nm}$ ) para $\mathrm{x}=0,14$. Coeficientes eletro-ópticos quadráticos tão altos quanto $17,5 \times 10^{-16} \mathrm{~m}^{2} / \mathrm{V}^{2}$ posicionam estes materiais como de melhor resposta electro-óptica entre os ferroelétricos.
\end{abstract}

Palavras-chave: cerâmicas de PLMN-PT, transmitância óptica, coeficientes eletroópticos.

\begin{abstract}
Lead magnesium niobate ceramics (PMN) belong to the class of ferroelectric materials used in various technological applications. However it is very difficult to produce a single phase material consisting of only the perovskite structure. Recent studies have shown that the addition of PT and La permit, respectively, to stabilize the perovskite phase and to achieve transparency of the order of commercial PLZT ceramics. In this work the powder synthesis of the system (1-x)PLMN-xPT as a function of PT (0.11 $\leq x \leq 0.15)$, for $1 \%$ mol of La, was carried out. Only traces of pyrochlore phase (2-3\%) in DRX powder were found, without a direct relationship with the concentration of PT. The results revealed a homogeneous microstructure, high density for all the compositions densified by hot pressing. High optical transmittance for $x=0.14$ was found $(55 \%$ in $\lambda=632 \mathrm{~nm})$. Quadratic electro-optic coefficients as high as $17.5 \times 10^{-16} \mathrm{~m}^{2} / V^{2}$ position these ceramics as the best response among the electro-optic ferroelectrics.
\end{abstract}

Keywords: PLMN-PT ceramics, optical transmittance, electro-optic coefficients.

\section{INTRODUÇÃO}

Niobato de magnésio e chumbo, $\mathrm{Pb}\left(\mathrm{Mg}_{1 / 3} \mathrm{Nb}_{2 / 3}\right) \mathrm{O}_{3}$ $(\mathrm{PMN})$, foi descoberto por Smolenskii e Agranovskaya enquanto estudavam um grande numero de compostos tipo perovskitas [1]. O PMN pertence à família de perovskitas complexas, sendo um importante material relaxor ferroelétrico com transição de fase difusa, elevados valores de coeficientes eletrostritivos e gigante valor de constante dielétrica, o qual faz deste material um importante candidato para a fabricação de capacitores multicamadas [2-4]. Além desta aplicação, o PMN possui diversas características que fazem dele um material adequado para dispositivos ópticos e biomédicos [5]. Contudo, as propriedades dielétricas, piezoelétricas e eletrostritivas do PMN podem ser melhoradas com a adição do titanato de chumbo (PT). A estabilização da fase perovskita também pode ser otimizada com essa adição, diminuindo assim a formação de fases parasitas (paraelétricas). No entanto, o aumento da concentração do PT promove uma diminuição gradual do comportamento relaxor do PMN, tornando a transição ferroelétrica mais normal [6].

Existem publicações que indicam a importância da adição de La em sistemas ferroelétricos, como o PZT, com o intuito de estudar as propriedades ópticas desses sistemas $[7,8]$. Sendo assim, pode-se considerar que a adição de $\mathrm{La}^{3+}$ no sistema PMN-PT pode potencializar suas propriedades ópticas e eletro-ópticas. Não obstante, as propriedades dielétricas das cerâmicas de PMN-PT podem ser fortemente prejudicadas por causa do aumento da formação da fase 
pirocloro ao adicionar $\mathrm{La}^{3+}$ ao sistema [9]. Um recente estudo realizado no GCFerr, permitiu concluir que a calcinação e densificação do sistema $\mathrm{Pb}, \mathrm{La}\left(\mathrm{Mg}_{1 / 3} \mathrm{Nb}_{2 / 3}\right) \mathrm{O}_{3}-\mathrm{PbTiO}_{3}$, PLMN-PT em atmosfera rica em oxigênio permite eliminar a formação de fases secundarias tipo pirocloro, causadas pela incorporação do La nas cerâmicas do sistema PMN-PT.

O sistema PLMN-PT é um sistema que pertence à classe de cerâmicas ferroelétricas transparentes (CFT). CFT constituem uma classe de materiais de incontestável relevância para a indústria eletro-eletrônica $[2,7]$. Um material eletro-óptico pode ser definido como um meio no qual uma informação elétrica pode ser transformada em informação óptica, e também no qual suas funções ópticas possam ser controladas através da aplicação de campos elétricos externos [10]. Entre as principais aplicações das CFT é possível citar: dispositivos de armazenamento de imagem (holografia), displays, moduladores e filtros opticamente ativos, sensores, etc [2]. Todas estas aplicações estão relacionadas principalmente com as propriedades ópticas e eletro-ópticas das CFT. Cerâmicas ferroelétricas transparentes podem apresentar tanto o efeito eletro-óptico linear (Pockels), como o efeito eletro-óptico quadrático (Kerr). No caso, de cerâmicas com estrutura cúbica ou pseudocúbica, sem polarização previa, o coeficiente Kerr prevalece [2].

Até agora não foi encontrado nenhum estudo que analize as propriedades ópticas e eletro-ópticas de cerâmicas de PLMN-PT em função da concentração de PT. Sendo assim, o objetivo deste trabalho é realizar um inédito estudo sobre as caracterizações microestrutural, estrutural, óptica, e eletro-óptica, das cerâmicas de $(1-\mathrm{x})\left[\mathrm{Pb}(1-\mathrm{y}) \mathrm{La}_{\mathrm{y}}\left(\mathrm{Mg}_{1 / 3} \mathrm{Nb}_{2 / 3}\right)\right.$ $\left.\mathrm{O}_{3}\right]-\mathrm{xPbTiO}_{3} \operatorname{com} 0,11 \leq \mathrm{x} \leq 0,15$ e $\mathrm{y}=0,01$, obtidas por prensagem uniaxial a quente.

\section{MATERIAIS E MÉTODOS}

As cerâmicas e os pós foram obtidos usando a fórmula: $(1-x)\left[\mathrm{Pb}_{(1-y)} \mathrm{La}_{\mathrm{y}}\left(\mathrm{Mg}_{1 / 3} \mathrm{Nb}_{2 / 3}\right) \mathrm{O}_{3}\right]-\mathrm{xPbTiO}_{3}$, com $0,11 \leq \mathrm{x} \leq$ 0,15 e y $=0,01$, denominados PLMN-xPT com $11 \leq x \leq 15$. A síntese dos pós foi realizada pelo método da columbita [11] usando óxido de lantânio, $\mathrm{La}_{2} \mathrm{O}_{3}$ (Aldrich, pureza $>99 \%$ ), óxido de nióbio, $\mathrm{Nb}_{2} \mathrm{O}_{5}$ (Alfa Aesar, 99,9+\%), óxido de chumbo, $\mathrm{PbO}$ (MGK, 99\%), e óxido de titânio $\mathrm{TiO}_{2}$ (Alfa Aesar, 99,8\%). Um primeiro tratamento térmico foi feito no hidróxido de carbonato de magnésio pentahidratado $\left(\mathrm{MgCO}_{3}\right) 4 \cdot \mathrm{Mg}(\mathrm{OH})_{2} .5 \mathrm{H}_{2} \mathrm{O}$ (Aldrich, $99 \%$ ) a $1100{ }^{\circ} \mathrm{C}$ com o objetivo de obter $\mathrm{MgO}$. A fase columbita $\left(\mathrm{MgNb}_{2} \mathrm{O}_{6}\right)$ foi obtida a partir da reação estequiométrica entre os pós de $\mathrm{MgO}$ e $\mathrm{Nb}_{2} \mathrm{O}_{5}$ a $1100{ }^{\circ} \mathrm{C}$ por $4 \mathrm{~h}$, ao ar. Seguidamente, $\mathrm{MgNb}_{2} \mathrm{O}_{6}$, (MN) foi moído em álcool isopropilico por 24

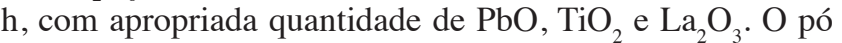
resultante foi secado e logo calcinado a $900{ }^{\circ} \mathrm{C}$ por $4 \mathrm{~h} \mathrm{em}$ um forno com controle de atmosfera a uma pressão de 200 $\mathrm{kPa}$ sob oxigênio.

Corpos cerâmicos transparentes de PLMN-xPT foram obtidos por meio de densificação por prensagem uniaxial a quente, em uma prensa Thermal Technology Inc. As amostras foram sinterizadas a $1250{ }^{\circ} \mathrm{C}$ por $4 \mathrm{~h}$ sob a ação de uma pressão uniaxial de 6,2 $\mathrm{MPa}$ em atmosfera parcial de $\mathrm{O}_{2}$. Análises por difração de raios $\mathrm{X}(\mathrm{DRX})$ e microscopia eletrônica de varredura (MEV) foram feitas tanto nos pós calcinados como nas cerâmicas densificadas. As cerâmicas foram cortadas e polidas opticamente com pasta de diamante de $3 \mu \mathrm{m}$ para as caracterizações ópticas, eletro-ópticas e microestruturais. Para estas últimas, as cerâmicas também foram submetidas a ataque térmico $\left(1150^{\circ} \mathrm{C}\right.$ por $\left.1 \mathrm{~min}\right)$. No caso das medidas eletro-ópticas, eletrodos de prata foram colocados nos lados laterais das cerâmicas, sendo estas medidas obtidas usando a técnica Senarmont [12].

\section{RESULTADOS E DISCUSSÃO}

Na Fig. 1 podem ser vistos os difratogramas de raios $\mathrm{X}$ obtidos na temperatura ambiente dos pós de PLMN-xPT com $11 \leq \mathrm{x} \leq 15$, calcinados a $900{ }^{\circ} \mathrm{C}$ por 4 h em oxigênio. É possível observar a formação de uma majoritária fase perovskita pseudo-cúbica (JCPDS 391488), já reportada [13, 14]. Fases secundarias tipo pirocloro $\mathrm{Pb}_{1,87}\left(\mathrm{Mg}_{0,29} \mathrm{Nb}_{1,71}\right) \mathrm{O}_{6,39}$ (JCPDS 37-0071) e não reagidas como $\mathrm{PbO}$ e $\mathrm{MgNb}_{2} \mathrm{O}_{6}$ também foram detectadas. Nenhuma relação entre concentração de PT e formação de fase foi possível deduzir a partir destes difratogramas.

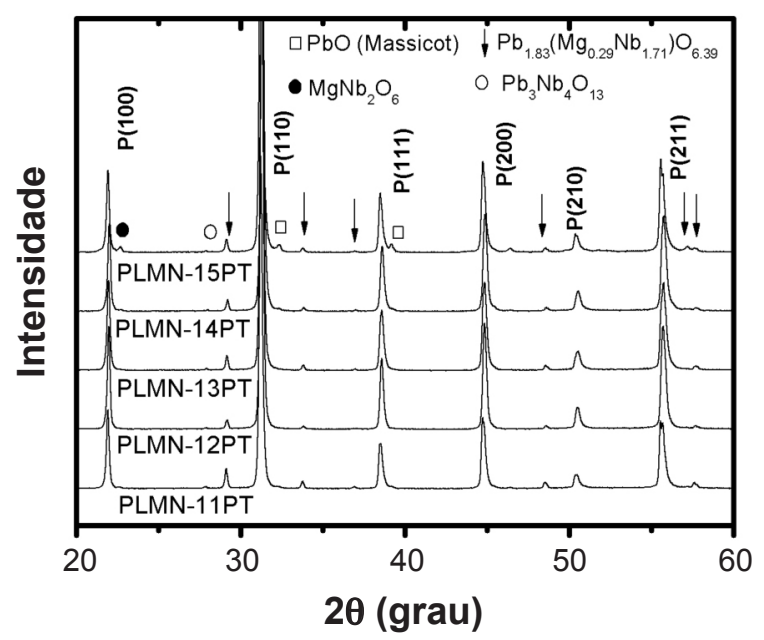

Figura 1: Difratogramas de raios $X$ e identificação das fases formadas nos pós de PLMN-xPT com $11 \leq \mathrm{x} \leq 15$, calcinados a $900{ }^{\circ} \mathrm{C}$ por $4 \mathrm{~h}$ em oxigênio.

[Figure 1: X-ray diffraction patterns and phase identification of $P L M N-P T$ powders with $11 \leq x \leq 15$, calcined at $900{ }^{\circ} \mathrm{C}$ for $4 \mathrm{~h}$ under oxygen.]

A Fig. 2 mostra imagens dos pós calcinados de PLMN$\mathrm{xPT}$ com $11 \leq \mathrm{x} \leq 15$. Pode-se observar a formação de aglomerados e partículas de forma irregular com tamanho aproximado de $1 \mu \mathrm{m}$ para todas as variações de concentração de PT. Tamanho de partícula similar foi reportado na preparação de pós de PMN por métodos químicos [15]. Contudo, não foi possível observar uma relação entre concentração do PT, formação de aglomerados, e tamanho de partícula. Fases secundárias não foram observadas nas 

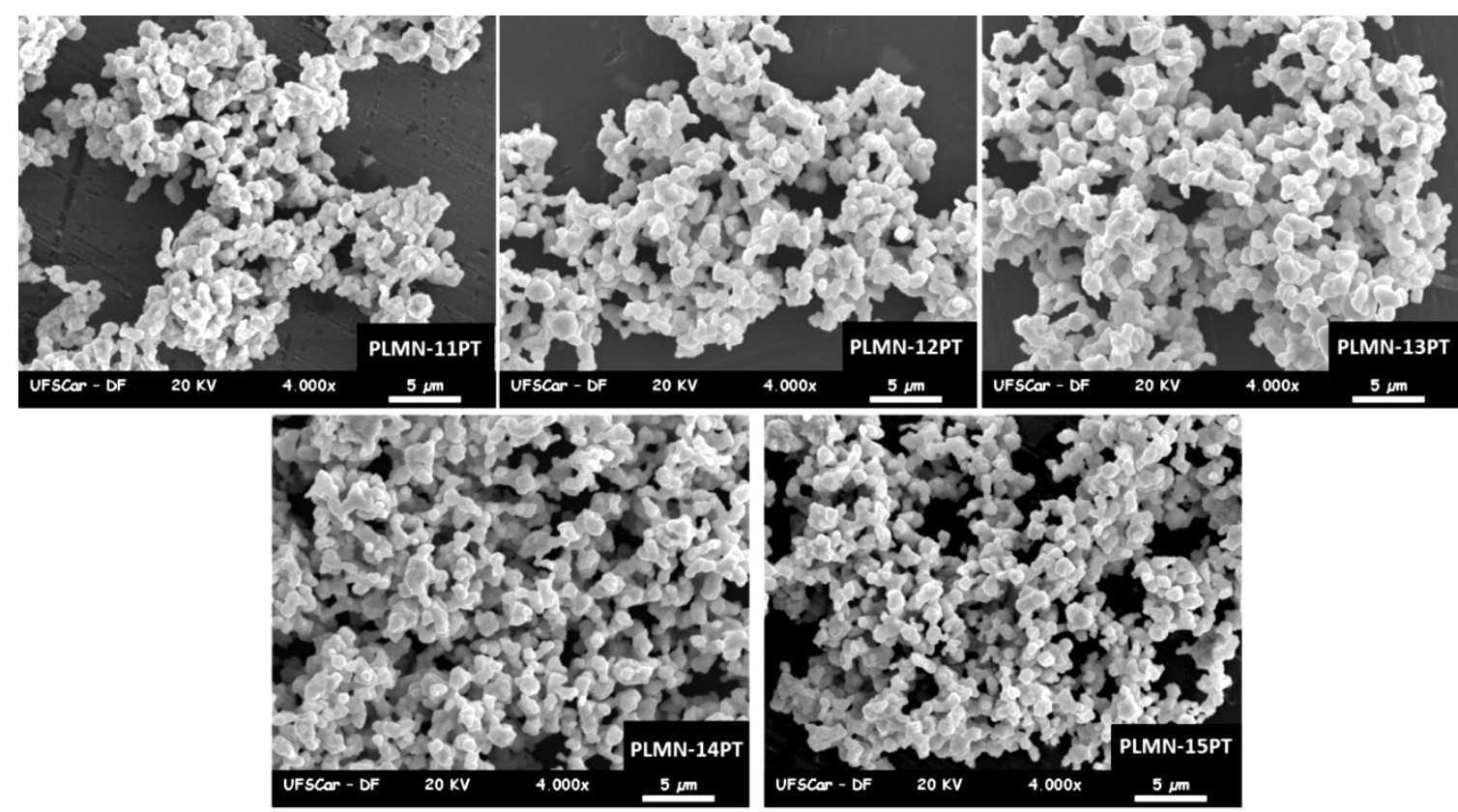

Figura 2: Imagens de microscopia eletrônica de varredura dos pós de PLMN-xPT, $11 \leq \mathrm{x} \leq 15$ calcinados a $900{ }^{\circ} \mathrm{C}$ por $4 \mathrm{~h}$ em oxigênio. [Figure 2: SEM images of PLMN-xPT powders, $11 \leq x \leq 15$, calcined at $900^{\circ} \mathrm{C}$ for 4 h under oxygen.]

micrografias BEI (não apresentadas aqui) realizadas nos pós de PLMN-xPT com $11 \leq x \leq 15$.

A Fig. 3 corresponde aos difratogramas de raios $X$ dos corpos cerâmicos de PLMN-xPT com $11 \leq \mathrm{x} \leq 15$ densificados por prensagem uniaxial a quente. Para todas as concentrações de PT se observou a formação da fase perovskita com simetria romboédrica (pseudo-cúbica, JCPDS 39-1488). Nenhuma fase secundaria foi observada nos diferentes difratogramas das cerâmicas neste trabalho, à exceção do difratograma correspondente à cerâmica PLMN15PT (aproximadamente $1 \%$ de fase pirocloro). Isto pode ser

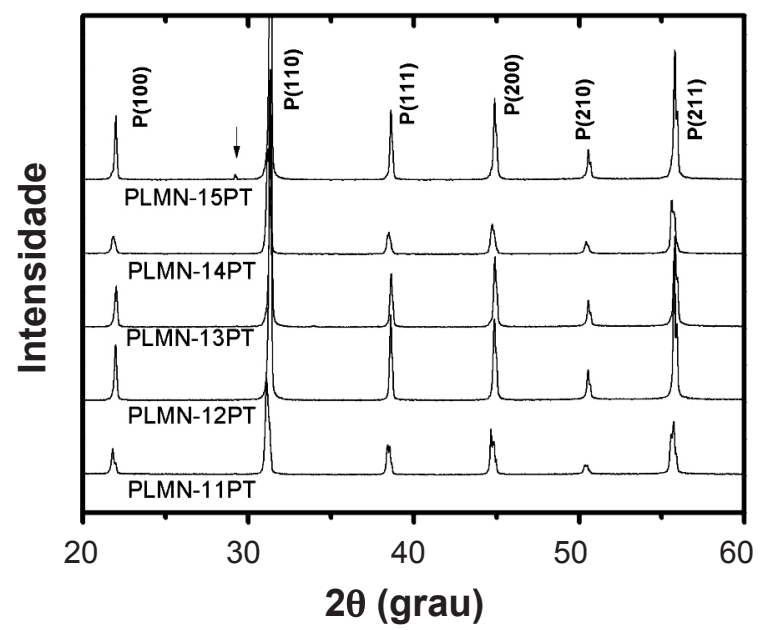

Figura 3: Difratogramas de raios X das cerâmicas de PLMN-xPT com $11 \leq \mathrm{x} \leq 15$, densificadas por prensagem uniaxial a quente a $1250{ }^{\circ} \mathrm{C}$ por $4 \mathrm{~h}$, sob oxigênio.

[Figure 3: X-ray diffraction patterns of PLMN-xPT ceramics with $11 \leq x \leq 15$ densified by uniaxial hot pressing to $1250{ }^{\circ} \mathrm{C}$, for $4 \mathrm{~h}$ under oxygen.] considerado como o primeiro indício da alta qualidade das amostras cerâmicas analisadas neste trabalho. Os parâmetros de rede, assim como as densidades teóricas (determinadas por meio dos difratogramas de raios $\mathrm{X}$ ), densidades aparentes relativas, e tamanho de grão estão listados na Tabela I. As amostras alcançaram densidades muito próximas à densidade ideal, confirmando a potencialidade do procedimento de prensagem uniaxial a quente empregado para a obtenção de corpos cerâmicos transparentes. Observa-se uma diminuição nos valores de densidade aparente e densidade teórica em função do aumento de PT, possivelmente causado por uma maior quantidade de PT nas amostras, permitindo uma maior volatilização deste composto, não obstante, este fato ainda não foi totalmente compreendido.

As micrografias das cerâmicas de PMN-11PT, PLMN-13PT e PLMN-15PT polidas e atacadas termicamente podem ser vistas na Fig. 4. Podem-se observar microestruturas uniformes, com grãos orientados

Tabela I - Parâmetros de rede (a), densidades teóricas $\left(\varrho_{t}\right)$, densidades aparentes relativas $\left(\varrho_{a}\right)$ e tamanho de grão $(\sigma)$ das cerâmicas de PLMN-PT.

[Table I - Lattice parameter (a), theoretical density $\left(\varrho_{P}\right)$, relative density $\left(\varrho_{a}\right)$ and grain size $(\sigma)$ of the PLMN-PT ceramics.]

\begin{tabular}{ccccc}
\hline Amostra & $\begin{array}{c}\mathrm{a} \\
(\AA)\end{array}$ & $\begin{array}{c}\varrho_{\mathrm{t}} \\
(\%)\end{array}$ & $\begin{array}{c}\varrho_{\mathrm{a}} \\
\left(\mathrm{g} / \mathrm{cm}^{3}\right)\end{array}$ & $\begin{array}{c}\sigma \\
(\mu \mathrm{m})\end{array}$ \\
\hline PLMN-11PT & 4,081 & 99,6 & $8,15 \pm 0,02$ & $9,06 \pm 1,10$ \\
PLMN-12PT & 4,037 & 99,6 & $8,15 \pm 0,02$ & $8,92 \pm 0,46$ \\
PLMN-13PT & 4,012 & 99,3 & $8,12 \pm 0,02$ & $8,54 \pm 0,56$ \\
PLMN-14PT & 4,010 & 99,0 & $8,10 \pm 0,02$ & $8,49 \pm 0,79$ \\
PLMN-15PT & 4,008 & 98,9 & $8,09 \pm 0,02$ & $8,51 \pm 0,47$ \\
\hline
\end{tabular}



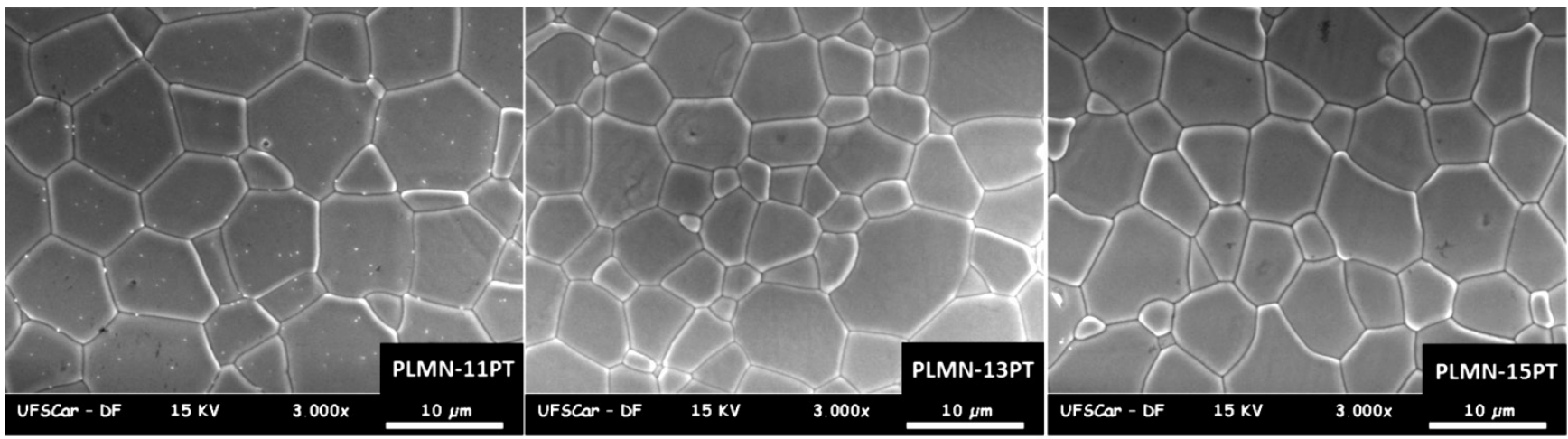

Figura 4: Micrografias (SEI) das cerâmicas PLMN-11PT, PLMN-13PT e PLMN-15PT, densificadas por prensagem uniaxial a quente a $1250{ }^{\circ} \mathrm{C}$ por $4 \mathrm{~h}$, em oxigênio.

[Figure 4: SEM images of PLMN-11PT, PLMN-13PT and PLMN-15PT ceramics, densified by uniaxial hot pressed to $1250{ }^{\circ} \mathrm{C}$, for $4 \mathrm{~h}$ under oxygen.]

aleatoriamente e praticamente sem porosidade. O tamanho médio de grão para as cerâmicas densificadas está listado na Tabela I. É possível verificar que o tamanho médio de grão tende a diminuir com o aumento da concentração de PT. Grãos com forma e tamanhos característicos de fase pirocloro não foram observados nessas micrografias.

A Fig. 5 mostra a fotografia de uma cerâmica ferroelétrica de PLMN-PT obtida por prensagem uniaxial a quente.

A Fig. 6 apresenta as curvas de transmitância, em função do comprimento de onda da luz incidente, na região do visível ao infra-vermelho próximo, para as cerâmicas de PLMN-PT, desenvolvidas neste trabalho. Nota-se um aumento na transmitância com o aumento do comprimento de onda para todas as cerâmicas, comportamento e valores de transmitância similares aos reportados em amostras de PLZT [16]. Destaca-se a maior transmitância da cerâmica PLMN14PT e a menor transmitância da amostra PLMN-11PT para comprimentos de onda maiores a $480 \mathrm{~nm}$. A transmitância das amostras PLMN-12PT e PLMN-13PT é praticamente

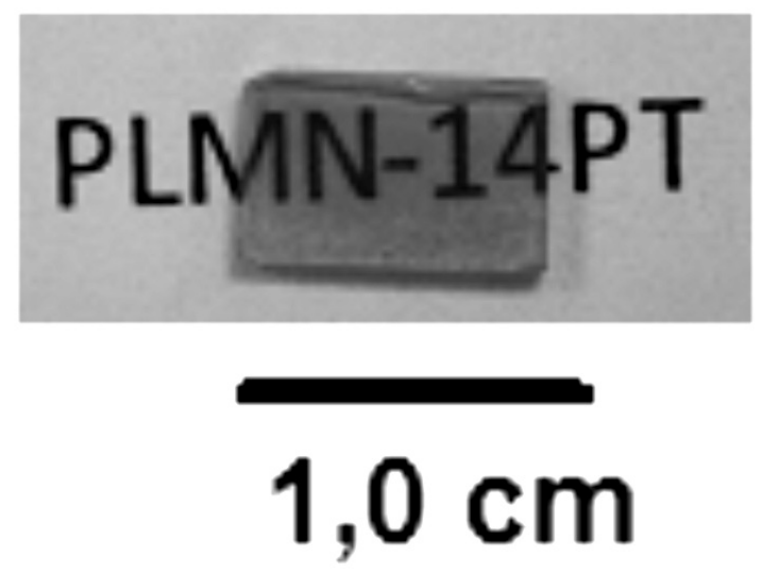

Figura 5: Exemplo típico de uma cerâmica ferroelétrica de PLMN$14 \mathrm{PT}$, polida oticamente após prensagem a quente, com espessura de $630 \mu \mathrm{m}$.

[Figure 5: Typical example of PLMN-14PT, ferroelectric ceramic, polished optically after uniaxial hot pressing, with a thickness of $630 \mu \mathrm{m}$.]

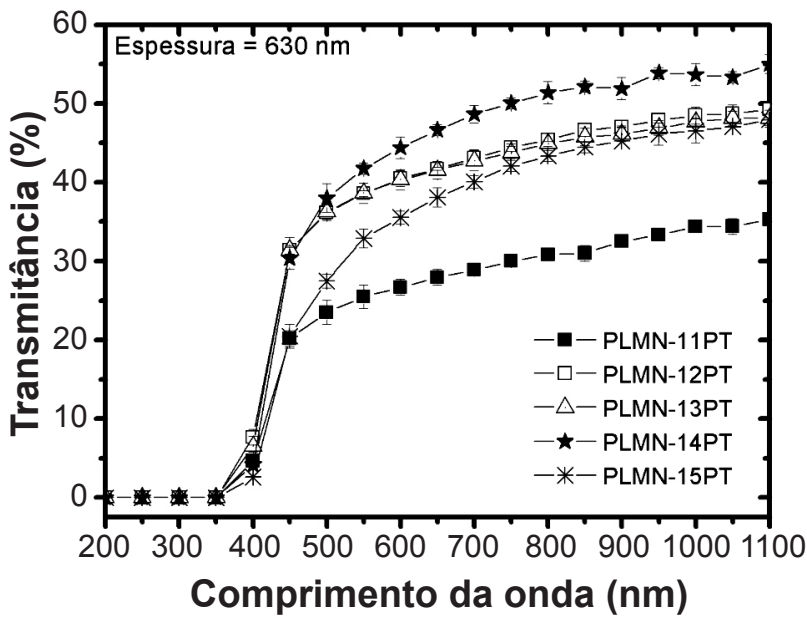

Figura 6: Transmitância em função do comprimento de onda da luz incidente, à temperatura ambiente, para PLMN-xPT com 11 $\leq \mathrm{x} \leq 15$, prensadas uniaxialmente a quente. Amostras polidas opticamente e com $630 \mu \mathrm{m}$ de espessura.

[Figure 6: Transmittance as a function of the wavelength of the incident light, at room temperature, of PLMN-xPT ceramics with $11 \leq x \leq 15$, after uniaxial hot pressing. Optical polishing, thickness $630 \mu \mathrm{m}$.]

igual. A diferença de transmitância entre estas amostras pode estar relacionada com maior redução de excesso de $\mathrm{PbO}$ nos contornos de grão em função do aumento da concentração do PT, como reportado em cerâmicas de PLT [17] (resultado coerente com a diminuição de densidade em função do aumento de PT); entretanto, isto não justificaria a menor transmitância da amostra PLMN-15PT. Sem dúvida, podese atribuir o comportamento da transmitância da cerâmica PLMN-15PT à possível aparição de centros espalhadores de origem intrínseca [17] (espalhamento por causa dos domínios ferroelétricos), que podem ser potencializados a partir dessa concentração de PT.

Medidas eletro-ópticas usando a técnica Senarmont [12] foram realizadas para todas as cerâmicas. A partir dessas medidas foi possível calcular o coeficiente eletroóptico quadrático, Kerr, para cada uma das concentrações de PT. Os valores destes coeficientes são apresentados na 
Tabela II - Coeficiente eletro-óptico quadrático Kerr das cerâmicas de PLMN-xPT com $11 \leq \mathrm{x} \leq 15$.

[Table II - Quadratic electro-optic coefficient (Kerr) for $P L M N-x P T$ ceramics with $11 \leq x \leq 15$.]

\begin{tabular}{cc}
\hline Amostra & Coeficiente Kerr $\times 10^{-16} \mathrm{~m}^{2} / \mathrm{V}^{2}$ \\
\hline PLMN-11PT & $6,2 \pm 0,2$ \\
PLMN-12PT & $17,5 \pm 0,4$ \\
PLMN-13PT & $20,7 \pm 0,9$ \\
PLMN-14PT & $10,8 \pm 0,2$ \\
PLMN-15PT & $10,8 \pm 0,3$ \\
\hline
\end{tabular}

Tabela II. A comparação destes resultados com aqueles reportados [18, 19] demonstram a alta qualidade das amostras obtidas, abrindo desta forma novas frentes de pesquisa e perspectivas com relação ao uso destas cerâmicas como parte de dispositivos eletro-ópticos. Comparando os resultados apresentados na Tabela II com os reportados é possível observar que as amostras de PLMN-PT densificadas neste trabalho apresentam coeficientes eletroópticos quadráticos da ordem dos apresentados pelo PLZT [18] e até aproximadamente duas vezes mais elevado que o apresentado nas cerâmicas comerciais de PLZT, quando comparadas com as cerâmicas PLMN-12PT e PLMN-13PT. Tal característica faz do PLMN-PT um material promissor como elemento ativo em dispositivos eletro-ópticos em substituição ao PLZT.

\section{CONCLUSÕES}

Foram apresentados resultados inéditos referentes à obtenção e caracterização de cerâmicas ferroelétricas transparentes de PLMN-xPT com $11 \leq \mathrm{x} \leq 15$. O emprego da técnica de densificação assistida por pressão uniaxial a quente possibilitou a obtenção de cerâmicas com ótimas propriedades microestruturais, estruturais, ópticas e eletro-ópticas em relação aos reportados. Cerâmicas do sistema PLMN-PT apresentaram características ópticas e eletro-ópticas que as fazem surgir como fortes candidatas a uma família de CFT, inclusive com coeficientes eletroópticos maiores aos apresentados pelas CFT de PLZT. A transmitância das cerâmicas de PLMN-PT prensadas uniaxialmente a quente é fortemente dependente da concentração de PT devido possivelmente à redução de fase de $\mathrm{PbO}$ nos contornos de grão, que não foi detectada pela técnica de difração de raios $\mathrm{X}$.

\section{AGRADECIMENTOS}

À CAPES, FAPESP, FUNDAYACUCHO e CNPq pelo suporte financeiro. À Dra. Y. P. Mascarenhas do Instituto de Física de S. Carlos, USP, pelo uso do laboratório de difração de raios X. Aos técnicos Natalia Zanardi e Francisco Jose Picon pelo suporte técnico.

\section{REFERÊNCIAS}

[1] F. S. Galassso, Structure, properties and preparation of perovskite, Pergamon Press, Oxford, Inglaterra (1969).

[2] K. Uchino, Ferroelectric Devices, Marcel Dekker, New York (2000).

[3] K. Uchino, Am. Ceram. Soc. Bull. 65 (1986) 647-652.

[4] S. Shannigrahi, K. Yao, J. Eur. Ceram. Soc. 25 (2005) 759-765.

[5] D. Mohan, R. Prasad, S. Banerjee, Ceram. Int. 27 (2001) 243-246.

[6] S. W. Choi, T. R. Shrout, S. J. Jang, A. S. Bhalla, Ferroelectrics 100 (1989) 29-38

[7] G. Haertling, J. Am. Ceram. Soc. 82, 4 (1999) 797-818.

[8] T. Comyn, A. Tavernor, J. Eur. Ceram. Soc. 20, 12 (2000) 2035-2038.

[9] S. M. Gupta, D. Viehland, J. Appl. Phys. 80, 10 (1996) 5875-5883.

[10] M. Levinson, Electronic Ceramics: Properties, Devices and Applications, Marcel Dekker Inc, New York, EUA (1984).

[11] L. Swartz, T. R. Shrout, Mater. Res. Bull. 17, 10 (1982) 1245-1250.

[12] M. Aillerie, M. D. Fontana, F. Abdi, C. CarabatosNedelec, N. Theofanous, J. Appl. Phys. 65, 6 (1989) 24062408.

[13] N. Wu, X. Song, Y. Hou, M. Zhu, C. Wang, H. Yan, Chinese Sci. Bull. 54, 7 (2009) 1267-1275.

[14] K. Kusumoto, T. Sekiya, Mater. Res. Bull. 33, 9 (1998) 1367-1375.

[15] P. K. Panda, B. Sahoo, Mater. Chem. Phys. 93, (2005) 231-236.

[16] Y. Abe, K. Kakegawa, J. Am. Ceram. Soc. 85, 2 (2002) 473-475.

[17] F. A. Londono, D. Garcia, J. A. Eiras, Rev. Latinoam. Metal. Mater. 31, 1 (2011) 52-58.

[18] K. Uchino, Ceram. Int. 21 (1995) 309-315.

[19] G. H. Haertling, C. E. Land, J. Am. Ceram. Soc. 54, 1 (1971) 1-11.

(Rec. 21/09/2010, Rev. 23/01/2011, Ac.05/02/2011) 